\title{
BMJ Open Point-of-care tests for urinary tract infections: protocol for a systematic review and meta-analysis of diagnostic test accuracy
}

\author{
David Fraile Navarro (D) , ${ }^{1}$ Frank Sullivan, ${ }^{1,2}$ Amaya Azcoaga-Lorenzo, ${ }^{1}$ \\ Virginia Hernandez Santiago (D) ${ }^{1}$
}

To cite: Fraile Navarro D, Sullivan F, Azcoaga-Lorenzo A, et al. Point-of-care tests for urinary tract infections: protocol for a systematic review and meta-analysis of diagnostic test accuracy. BMJ Open 2020;10:e033424. doi:10.1136/ bmjopen-2019-033424

- Prepublication history and additional material for this paper are available online. To view these files, please visit the journal online (http://dx.doi. org/10.1136/bmjopen-2019033424).

Received 05 August 2019 Revised 14 April 2020 Accepted 07 May 2020

\section{Check for updates}

\section{(c) Author(s) (or their} employer(s)) 2020. Re-use permitted under CC BY-NC. No commercial re-use. See rights and permissions. Published by BMJ.

${ }^{1}$ Division of Population and Behavioural Sciences, School of Medicine, University of Saint Andrews, Saint Andrews, UK

${ }^{2}$ Department of Family and Community Medicine, University of Toronto, Toronto, Ontario, Canada

Correspondence to Dr Virginia Hernandez Santiago; vhs2@st-andrews.ac.uk

\section{ABSTRACT}

Introduction Urinary tract infections (UTIS) are the second most common type of infection worldwide, accounting for a large number of primary care consultations and antibiotic prescribing. Current diagnosis is based on an empirical approach, relying on symptoms and occasional use of urine dipsticks. The diagnostic reference standard is still urine culture, although it is not routinely recommended for uncomplicated UTIs in the community, due to time to diagnosis (48 hours). Faster point-of-care tests have been developed, but their diagnostic accuracy has not been compared. Our objective is to systematically review and meta-analyse the diagnostic accuracy of currently available point-of-care tests for UTIs.

Methods and analysis Studies evaluating the diagnostic accuracy of point-of-care tests for UTIs will be included.

PubMed, Web of Science, Embase and Cochrane Database of Systematic Reviews were searched from inception to 1 June 2019. Data extraction and risk-of-bias assessment will be assessed using the Quality Assessment of Diagnostic Accuracy Studies tool. Meta-analysis will be performed depending on data availability and heterogeneity.

Ethics and dissemination This is a systematic review protocol and therefore formal ethical approval is not required, as no primary, identifiable, personal data will be collected. Patients or the public were not involved in the design of our research. However, the findings from this review will be shared with key stakeholders, including patient groups, clinicians and guideline developers, and will also be presented and national and international conferences.

PROSPERO registration number CRD42018112019.

\section{INTRODUCTION}

Urinary tract infections (UTIs) are the second most common cause of infection in primary ${ }^{1}$ and secondary care ${ }^{2}$ and most women experience at least one episode of acute uncomplicated cystitis in their lifetime. In the UK, UTIs account for $1 \%-3 \%$ of all consultations in primary care each year. ${ }^{1}$ UTIs are also responsible for a major part of antibiotic prescriptions, accounting for up to
Strengths and limitations of this study

- To our knowledge, this is the first systematic review that formally analyses and compares the diagnostic accuracy of currently available point-of-care tests for urinary tract infections (UTIs) diagnosis.

- The results from this study will help identify the best available point-of-care test to diagnose UTIs in the primary care setting.

- It is likely that significant heterogeneity will be found as all available tests will be explored, and metaanalysis will be performed to account for this.

- Studies involving paediatric population $(<18$ years old) or populations with certain conditions (detailed in exclusion criteria) will be excluded, as well as studies aimed at screening of asymptomatic bacteriuria, which may affect the generalisability of the results out with these situations.

$15 \%$ of antimicrobial use in the community, with antibiotic use been described as one of the main factors contributing to the emergence of antimicrobial resistance (AMR). ${ }^{3}$ The rise of AMR has been postulated as one of the major challenges for healthcare worldwide. ${ }^{4}$ It is related to increased morbidity, mortality and cost, particularly in vulnerable populations such as the elderly. ${ }^{4}$ The WHO Global Action Plan to Reduce Antimicrobial Resistance $^{5}$ includes improving antimicrobial use across all human and animal health, and environment settings through a One Health approach. Rates of AMR among gramnegative bacteria have progressively increased in the last decade in the European Union (EU) ${ }^{6}$ with particularly concerning rise of carbapenemase-producing and extendedspectrum-beta-lactamase organisms. ${ }^{7}$

Currently, most clinical guidelines recommend that primary care diagnosis and management of uncomplicated UTI should be done empirically, ${ }^{89}$ thus based on clinical 
symptoms. Although this approach has proven to be cost effective, ${ }^{10}$ prescribing without diagnostic certainty increases the use of potentially unnecessary antibiotics, and contributes to the problem of AMR. ${ }^{11}$ Up to $90 \%$ of patients presenting to primary care with urinary symptoms receive an antibiotic ${ }^{7}{ }^{12}$ but it is usually without further investigation, so it is unclear how many will have a proven infection. Available evidence on how well symptoms predict the presence of a true UTI has shown diverging results, when compared with gold standard (urine culture). The probability of a female patient presenting to primary care with typical UTI symptoms and having a confirmed infection is estimated to be between $50 \%-80 \%$, with the greatest predictability for haematuria, and if combined with a positive urine dipstick. ${ }^{13}{ }^{14}$ Therefore, alternative tests with enhanced diagnostic accuracy could potentially reduce inappropriate antimicrobial use in this context.

The gold standard for UTI diagnosis is urine culture from a midstream, clean urine catch, but as previously mentioned, urine culture is not always performed, especially in primary care and emergency departments, where diagnosis of most UTIs occurs. ${ }^{15}$ Urine culture is slow, requiring at least $24-48$ hours to report the causative microorganism and provide an antibiotic resistance profile, ${ }^{16}$ and symptoms are usually distressing enough to prompt on the day empirical management, since acutely unwell patients with UTI symptoms may not be prepared to wait up to 48 hours for a culture result. Current clinical guidelines also advocate empirical treatment if symptoms are sufficiently suggestive of a diagnosis of UTI. ${ }^{9}$ Empirical decision-making will often result in the patient getting an antibiotic without infection confirmation. As result of this, different point-of-care tests (POCT) have been developed aiming to provide a more rapid and accurate method for detecting infection. POCT has been defined as 'a test to support clinical decision making, performed nearby the patient and on any part of the patient's body or its derivates, to help the patient and healthcare professional on the best management approach during or very close to the time of the consultation, with results available at the time of clinical decision making. ${ }^{17}$ POCT diagnostic accuracy is influenced dramatically from pretest probability in different subpopulations ${ }^{18}$ and consequently, its ability to detect or discard infection can be variable. ${ }^{1920}$ Potentially, an 'ideal' POCT would allow for more timely identification of UTIs, facilitating improved, targeted treatment, and reduced inappropriate antibiotic use. Indirect methods, such as urine dipsticks, which detect host inflammatory response rather than bacterial presence, have become the main POCT for UTIs. ${ }^{21}$ Other techniques include culture-based devices, enzymatic assays and semiautomated urine analysers. ${ }^{22}$ Previous reports suggest that their diagnostic accuracy could be greater than that of simpler urine dipsticks. ${ }^{16}$ These tests could provide relevant information to clinicians to prescribe antimicrobials more accurately, reducing antibiotic-related harms (including resistance),

\begin{tabular}{ll}
\hline Table 1 Inclusion criteria & \\
\hline Participants: & $\begin{array}{l}\text { Adults } \\
\text { Presentation: }\end{array}$ \\
$\begin{array}{l}\text { Symptomatic UTIs (variously } \\
\text { defined) }{ }^{13}\end{array}$ \\
Index test(s): & $\begin{array}{l}\text { Any point-of-care diagnostic } \\
\text { test }\end{array}$ \\
Target condition: & Urinary tract infections \\
Reference standard: & Urine culture \\
\hline
\end{tabular}

UTIs, urinary tract infections.

and costs. ${ }^{23}$ However, it is difficult to ascertain which POCT could be better for diagnosing UTIs, in general, and in specific situations.

Our aim is to systematically review and meta-analyse the diagnostic test accuracy of currently available POCTs for UTIs, as compared with gold standard (urine culture).

\section{METHODS AND ANALYSIS}

\section{Eligibility criteria}

Randomised clinical trials (RCTs), cluster RCTs, evaluation studies, observational studies and regulatory or approval evidence reports (if available), evaluating point-of-care diagnostic tests for UTI in symptomatic patients versus urine culture (reference standard) ${ }^{7}$ will be included, from both primary or secondary care settings. No particular index test was prespecified in our review search criteria, as we aimed to capture and compare all available tests. However, only those tests that could be categorised as 'point-of-care test' will be included, defined as above. ${ }^{17}$ Inclusion criteria are detailed in table 1 , following the PIRD (Participants, Index test, Target condition, Reference standard) approach for including studies in systematic reviews of diagnostic test accuracy. ${ }^{24}$

The search strategy uses broad terms for defining UTI with the aim of capturing all potentially relevant studies looking at POCT used in symptomatic UTIs. Classical symptoms include those mentioned in table 1 , and we will also examine different symptoms/combinations and UTI definition used in each study. Exclusion criteria will be applied and are detailed below. These include:

- Studies evaluating the detection of asymptomatic bacteriuria in pregnancy.

- Studies performed only in children.

- Tests aimed at detecting sexually transmitted infections, or non-bacterial infections (eg, schistosomiasis).

- Tests based on biomarkers needing laboratory facilities.

- Studies whose main outcome measure is to detect complications of urinary infections (eg, CT scans or other imaging techniques).

- Clinical algorithms or self-reported symptom tests.

- Specific populations will be excluded: urinary catheterised patients, kidney transplantation, terminal 
kidney failure or immunocompromised patients, patients with spinal cord injury or neurogenic bladder.

\section{Information sources and search}

Medline, Web of Science, Embase and Cochrane Database of Systematic Reviews were searched from database inception to 1 June 2019 with no language restrictions. Only studies involving human health were included. The search included a combination of the following terms: "Urinary Tract Infections/diagnosis", "Diagnostic Tests, Routine", "Point-of-Care Systems", "Point-of-Care Testing", "point-of-care testing", 'near-patient testing', 'RDT', "poct", "Diagnostic Technics and Procedures", "Techni* and Procedures, Diagnostic", "rapid diagnostic test*", AND "Urinary Tract Infections", "Pyuria", "Bacteriuria", "uti", AND "sensitivity", "specificity", "likelihood ratio", "predictive value", "diagnostic accuracy", "AUC", "PPV", "NPV", among other. The full search strategy is available in online supplementary appendix 1 and online in PROSPERO's database. ${ }^{25}$

\section{Data management}

Search results will be stored in EndNote V.X8.2 bibliography management software. To synthetise and develop study selection, data extraction and quality assessment, we will use Covidence platform. ${ }^{26}$

\section{Study selection}

Three reviewers (DFN, AAL and VHS) will independently assess study eligibility for inclusion. A calibration exercise assessing $10 \%$ of the results by title and abstract will be done in duplicate. After title and abstract screening, selected articles will be screened full text. Discrepancies will be solved by discussion. Another reviewer will be involved as necessary (FS).

\section{Data collection process}

A standardised data extraction form will be developed. The review team (DFN, AAL and VHS) will independently extract the data from all studies. Study authors will be contacted if no data are available. All articles will be double extracted, and risk of bias will be double assessed. Discrepancies will be evaluated and solved by discussion, and if no agreement, a third reviewer will be involved.

\section{Diagnostic accuracy measures}

A $2 \times 2$ contingency table with true positives, true negatives, false positives and false negatives will be extracted from each study. Accuracy outcome measures will include: sensitivity, specificity, positive and negative predictive values.

\section{Definitions for data extraction}

From each study, besides the accuracy-related data already specified, we will extract the following predefined set of characteristics:

- Device/product name.

- Manufacturer/country of origin.

- Regulatory approval status in the EU and USA.
- Type of sample used (clean urine midstream catch, or other).

- Method principle (culture-based, enzymatic assay, other).

- Analysis time (time required, in minutes).

- Additional training required.

- Need for supplementary equipment (eg, steriliser, centrifuge, etc).

- Cost.

- Type of result provided if the test is positive (presence of infection, bacterial load, antibiotic sensitivity, indirect method for detection).

- The threshold for positivity detection, in unit forming colonies.

- Population tested.

- UTI definition used.

- Secondary outcomes: mortality, hospitalisation, quality of life measures and/or patients' preferences, if reported.

\section{Risk of bias}

Methodological quality assessment will be conducted using the Quality Assessment of Diagnostic Accuracy Studies tool. ${ }^{27}$

\section{Synthesis of results}

A narrative description summarising the prespecified characteristics of each test, and a paired sensitivity-specificity forest plot, will be provided. Meta-analysis will be performed depending on available data, sources of heterogeneity, comparability between methods and ability to aggregate data. If enough data are available, randomeffect meta-analysis will be performed for each index test. The bivariate model will be used to ascertain summary sensitivity and specificity if all studies in the group use the same threshold value for positivity. If index tests use different threshold values, the hierarchical summary receiver operating characteristic (HSROC) model will be used instead, to obtain summary sensitivity and specificity for each threshold value. Sources of heterogeneity will be investigated, regarding index test used, threshold for detection, target population included in the study and its given (if reported) pretest probability. Subgroup analysis will be explored and performed depending on the heterogeneity found and available data, analysing separately studies looking at each POCT and also different population groups (differentiating adults from elderly patients).

\section{Patients and public involvement}

Patients or the public were not involved in the design of our research. However, the findings from this review will be shared with key stakeholders, including patient groups, clinicians and guideline developers, and will also be presented and national and international conferences. 


\section{ETHICS AND DISSEMINATION}

Ethical approval was explored with the University of St Andrews School of Medicine Research and Ethics Committee but was not necessary due to the nature of the research (literature review). Results from this review will be shared with key stakeholders, including patient groups, clinicians and guideline developers, and will potentially inform future diagnostic and treatment pathways.

Contributors VHS conceived the idea. The protocol was developed by DFN, FS, AAL and VHS. DFN performed the search strategy. VHS, DFN and AAL will contribute to design the data extraction form, screen manuscripts, extract data from individual studies and assess study quality. FS will act as a third reviewer in case of discrepancy. DFN redacted the original draft of the protocol. All authors reviewed and contributed to subsequent drafts and read and approved the final draft.

Funding DFN is supported by an NES general practice academic fellowship, AAL is supported by an HDR UK clinical postdoctoral fellowship and VHS is supported by an NRS clinical academic fellowship.

Competing interests None declared.

Patient and public involvement Patients and/or the public were not involved in the design, or conduct, or reporting, or dissemination plans of this research.

Patient consent for publication Not required.

Provenance and peer review Not commissioned; externally peer reviewed.

Open access This is an open access article distributed in accordance with the Creative Commons Attribution Non Commercial (CC BY-NC 4.0) license, which permits others to distribute, remix, adapt, build upon this work non-commercially, and license their derivative works on different terms, provided the original work is properly cited, appropriate credit is given, any changes made indicated, and the use is non-commercial. See: http://creativecommons.org/licenses/by-nc/4.0/.

\section{ORCID iDs}

David Fraile Navarro http://orcid.org/0000-0002-1108-7071

Virginia Hernandez Santiago http://orcid.org/0000-0002-8544-1483

\section{REFERENCES}

1 National Prescribing Centre. Urinary tract infection MeReC Bulletin volume 17 number 3, 2006. Available: http://www.spitalmures.ro/_ files/protocoale_terapeutice/medicina de familie/acute_uti_final.pdf

2 Lyytikäinen O, Kanerva M, Agthe N, et al. Healthcare-associated infections in finnish acute care hospitals: a national prevalence survey, 2005. J Hosp Infect 2008;69:288-94.

3 McClean P, Hughes C, Tunney M, Gilpin D, et al. Antimicrobial prescribing in European nursing homes. J Antimicrob Chemother 2011;66:1609-16.

4 Levy SB, Marshall B. Antibacterial resistance worldwide: causes, challenges and responses. Nat Med 2004;10:S122-9.

5 WHO. Global action plan on antimicrobial resistance, 2017. Available: http://www.who.int/antimicrobial-resistance/publications/globalaction-plan/en/\#.W-GmsnwmOnA.mendeley [Accessed 6 Nov 2018]

6 Ciorba V, Odone A, Veronesi L, et al. Antibiotic resistance as a major public health concern: epidemiology and economic impact. Ann Ig 2015;27:562-79.

7 Scottish Intercollegiate Guidelines Network. Scottish intercollegiate guidelines network. Management of suspected bacterial urinary tract infection in adults : a national clinical guideline, 2012. Available: https://www.sign.ac.uk/sign-88-management-of-suspectedbacterial-urinary-tract-infection-in-adults.html [Accessed 2 Nov 2018].

8 Steadman S, Ahmed I, McGarry K, et al. Is screening for urine infection in well infants with prolonged jaundice required? local review and meta-analysis of existing data. Arch Dis Child 2016;101:614-9.

9 National Institute for Healthcare Excellence (NICE). Urinary tract infection (lower): antimicrobial prescribing guidance. Available: https://www.nice.org.uk/guidance/ng109\#.XdZwkGqjTpQ.mendeley [Accessed 21 Nov 2019].

10 Fenwick EA, Briggs $\mathrm{AH}$, Hawke $\mathrm{Cl}$. Management of urinary tract infection in general practice: a cost-effectiveness analysis. Br J Gen Pract 2000;50:635-9.

11 Hernandez-Santiago V, Davey PG, Nathwani D, et al. Changes in resistance among coliform bacteraemia associated with a primary care antimicrobial stewardship intervention: a population-based interrupted time series study. PLoS Med 2019;16:e1002825.

12 Fahey T, Webb E, Montgomery AA, et al. Clinical management of urinary tract infection in women: a prospective cohort study. Fam Pract 2003;20:1-6.

13 Bent S, Nallamothu BK, Simel DL, et al. Does this woman have an acute uncomplicated urinary tract infection? JAMA 2002;287:2701-10.

14 Giesen LGM, Cousins G, Dimitrov BD, et al. Predicting acute uncomplicated urinary tract infection in women: a systematic review of the diagnostic accuracy of symptoms and signs. BMC Fam Pract 2010;11:78.

15 Schmiemann G, Kniehl E, Gebhardt K, et al. The diagnosis of urinary tract infection: a systematic review. Dtsch Arztebl Int 2010;107:361-7.

16 Baker C. Accident and emergency statistics: demand, performance and pressure. House Commons Libr 2017;6964:1-32.

17 Schols AMR, Dinant G-J, Hopstaken R, et al. International definition of a point-of-care test in family practice: a modified e-Delphi procedure. Fam Pract 2018;35:475-80.

18 AGM, MDSD, TLF. Using urine dipstick as a presumptive result for urinary tract infection. Clin Chem 2015;61:S196.

19 Hurlbut TA, Littenberg B. The diagnostic accuracy of rapid dipstick tests to predict urinary tract infection. Am J Clin Pathol 1991;96:582-8.

20 WHO. Global antimicrobial resistance surveillance system (GLASS) report, 2018. Available: http://www.who.int/glass/resources/ publications/early-implementation-report/en/\#.W-K8qcwb57U. mendeley [Accessed 7 Nov 2018].

21 Little P, Moore MV, Turner S, et al. Effectiveness of five different approaches in management of urinary tract infection: randomised controlled trial. BMJ 2010;340:c199.

22 NIHR Diagnostic Evidence Co-operative Oxford. Point-of-care testing for urinary tract infections. horizon scan report. UK: Oxford, 2016.

23 Roberts RR, Hota B, Ahmad I, et al. Hospital and societal costs of antimicrobial-resistant infections in a Chicago teaching hospital: implications for antibiotic stewardship. Clin Infect Dis 2009:49:1175-84.

24 Leeflang MMG, Deeks JJ, Gatsonis C, et al. Systematic reviews of diagnostic test accuracy. Ann Intern Med 2008;149:889-97.

25 Fraile Navarro D, Sullivan F, Hernandez-Santiago V. Search Strategy for "Point-of-care tests for urinary tract infections: diagnostic test accuracy systematic review and meta-analysis", 2018. Available: https://www.crd.york.ac.uk/PROSPEROFILES/112019_STRATEGY_ 20181113.pdf [Accessed 17 Dec 2018].

26 Veritas Health Innovation. Covidence systematic review software. Available: www.covidence.org

27 Whiting PF, Rutjes AWS, Westwood ME, et al. QUADAS-2: a revised tool for the quality assessment of diagnostic accuracy studies. Ann Intern Med 2011;155:529. 